\title{
LOS VERSOS DE PRUDENCIO SOBRE EL EMPERADOR JULIANO
}

I'rud., I.ih. Apotheosis, vv. 419-154 is undoubtely the most ol,jective and trustworthy of the cliristian panegyrics on the Emperor Julian, and there are strong rcasons to suppose that Prudence is here following pagan literary sources, perhaps Eutropius or Ammianus. In vv. 455-502 Prudence transfers a previous literary topic (cf. Lact., De mortibus, X 2,4) for his own purposes in order to illustrate Julian's paganism. Other minor remarks on the text are done.

La impresión que produjo en sus contemporáneos el breve reinado del Emperador Juliano (desde noviembre del 361 hasta junio del 363 como Augusto único) fue extraordinaria. Paganos y cristianos se aprestaron a escribir sobre él, unos en decidida defensa de su persona y de sus acciones, y otros utilizando las más duras e incluso denigrantes invectivas. Ejemplo de ambas posturas serían, por un lado, la del so-

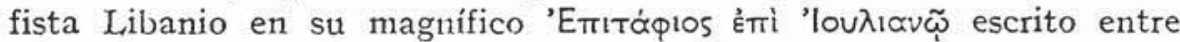

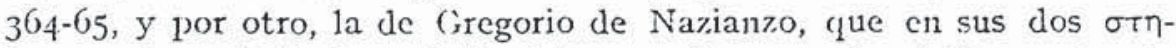

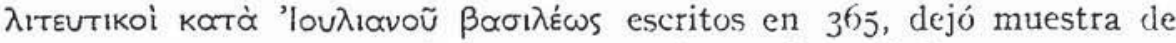
uno de los ataques más violentos que se hayan formulado nunca contra el Emperador. La trágica muerte en Persia de Juliano y la misma magna campaña, aumentaron incluso este interés, y pronto surgieron multitud de relatos más o menos exactos o confusos sobre el Emperador. E1 mundo bizantino, impresionado por la calidad literaria e intelectual de Juliano, guardó siempre recuerdo de él, y los escritores e historiadores cristianos aprovecharon la oportunidad que les brindaba la figura del Emperador que había querido volver al culto de los antiguos dioses para polemizar con sus escritos (tal es el caso de Cirilo de Alejandría) o para hacer propaganda de la vieja idea cristiana de que el que se alza contra Dios perecerá. La leyenda se apoderó rápidamente de él llegando hasta los más recónditos lugares el relato mixtificado de sus hechos. Así, por ejemplo, es posible leer en la Crónica de Juan, obispo de Nikiou, escrita en griego originalmente en la segunda mitad del siglo vir, frases como ésta: "Semejante a Satán, su padre, este Emperador tiránico destruyó los edificios sagrados fundados por el Emperador

9 
Constantino, el amigo de Dios, y transformó los edificios en casa de demonios y en templos de los ídolos» ${ }^{1}$. La complicación de estas "historietas" llega a extremos insospechados como lo prueban bien palmariamente estos comentarios del escoliasta al "Paraclitus» de Warnerius. Basiliensis, escritos en el siglo $\mathrm{xr}$ :

Hic autor ponit aliud exemplum de Juliano qui adiutorio Sathana effectus fuit papa Katholicus et oculte destruebat ecclesiam et a cardinalibus fuit repulsus de papatu; qui iterum vigore diabolico fuit in Cesarem electus et ultore Mercurio cardinalli gladio est interfectus ${ }^{2}$.

1 Cf. Chronique de Jean, evêque de Nikiou. Texte ethiopien, tr. par H. Zottemberg, Paris, 1883 , cap. LXXVII. La crónica de Juan de Nikiou nos ha llegado a través de una versión etiópica, hecha sobre una antigua paráfrasis árabe en 1062 por un sabio abisinio de nombre desconocido y un monje egipcio llamado Gabriel, su colaborador. El texto fue escrito originalnente en griego y algunos capitulos lo fueron en copto debido al tipo de fuentes que utiliza el cronista. Cf. Zottemberg, pp. 5-7.

2 Schol. ad v. $47^{8}$ del Paraclitus; cf. P. Thomas, Annales de l'Institut de philologie et d'Histoire Orientales, 2, 1934, p. 453. Historias curiosas sobre Juliano se pueden leer en la crónica de Al-Tabari, tr. por T. Nöldeke en Geschichte der Perser und Araber zur Zeit der Sasaniden aus der arabischen Chronik des Tabari übersetzt, Leiden, 1879 (ed. fotomecánica, Graz, 1973); en Ta'Alibi en su Histoire des Rois des Perses, trad. H. Zottemberg, San Petersburgo, 1900; en el PseudoMoises de Chorene, Histoire de l'A rmenie, tr. V. Langlois para la Collection des historiens anciens et modernes de l'Armenie, II, Paris, 1860 ), o en el historiador armenio Fausto de Buzanta (cf. FHG, V, ed. Müller). Para un poema en sirio sobre Juliano cf. $\mathrm{H}$. Gollancz, Julian the Apostate now translated for the first time from the Syriac original, London, 1928; buen estudio y abundante bibliografia sobre estas leyendas (ciclo de S. Mercurio, etc.) en N. Baynes, "The Death of Julian the Apostate in a Christian Legend", JRS, 27, 1937, p. 22 ss.; del mismo autor cf. "Rome and Armenia in the Fourth Centuryn, EHR, 25, 1910, pp. 625-643 reproducido en sus Byzantine Studies, London, 1955; curiosa reminiscencia de estas leyendas que en forma de canciones populares se conservan aún en nuestros días, en R. M. Dawkins, "A Byzantine Carol in Honour of St. Basil", JIIS, 66, 1946, p. 43 ss.; para estas historias en Europa cf. A. J. Denomy, "An Old French Version of the Julian Episode in the Life of St. Basil, MST, I8, 1950, p. 105 ss. Lin Lispaña la leyenda se transmitió también $y$ aparece en un episodio de las Cantigas de Alfonso el Sabio (cf. ed. de la Real Academia de la Historia, Madricl, $1 \mathrm{SS}_{9}$, p. 23 ss., citado en Denomy, n. 4). Indice curioso de la fama y difusión de la leyenda de Juliano la tenemos en el hecho de que en la igiesia de (Q'asr Ibrim en Nubia habia escritas en el pavimento homilias sobre la leyenda de $\mathrm{S}$. Mercurio y el juicio de Dios sobre Juliano (cf. W. H. C. Frend, Martyrdom and Persecution in the Early Church, Oxford, I965, p. IX y n. 3). Un buen resumen de la cuestión puede leerse en la inmejorable obra de J. Bidez, La Vie de l'Empereur Iutien, 2. ${ }^{\text {a }}$ ed. Paris, 1965. página 332 ss. y 338 ss. En época moderna, sin mencionar a Lorenzo de Medicis ni a Voltaire, es posible rastrear aún la preocupación de novelistas y escritores 
Parecidas historias corrían ya en épocas del mismo Juliano como lo demuestra el texto de Prudencio que va a ser objeto de nuestro comentario en este trabajo. Este texto al que con cierta frecuencia se alude no ha sido aún estudiado sistemáticamente ni valorado en su conjunto.

A. Prudencio Clemente, nacido en 348 en Hispania Tarraconnensis muy probablemente ${ }^{1}$, fue gobernador de una provincia (quizás Hispania) y estuvo unido a un César, obteniendo el cargo de comes primis ordinis $^{2}$. Como ya es sabido no parece que escribiera nada antes del 392 y su actividad literaria puede situarse entre 395-405. E1 pasaje en el que se encuentra la alusión al Emperador Juliano se halla en el Liber Apotheosis, escrito hacia el año $400^{3}$, que es un conjunto de hexámetros de carácter didáctico destinado a combatir las herejías ${ }^{4}$. Hacia la mitad aproximadamente del poema Prudencio dice:

sobre Juliano (recuérdense a este respecto el drama de Ibsen, Emperador y Galileo, la novela del ruso Merekowsky, La muerte de los dioses o simplemente la deliciosa novela de Core Vidal. Juliano el Apóstata).

1 Cf. Praef. 24-25 (seguimos la ed. del Corpus Christianornti, CXXVI, de M. P. Cunningham). Sobre su carrera politica cf. Jones-Martindale-2Iorris, The Prosopography of the Later Roman Empire, Cambridge, 1971, I, p. 214. No vamos a entrar aqui en la discusión de si era natural de Calagurris o de Caesaraugusta. Sólo diremos que los argumentos normalmente aducidos para una y otra alternativa - Perist. IV 3 I (nostra Calagurris) y Perist. IV I (noster populus hablando de Caesaraugusta), etc. - no nos parecen totalmente convincentes desde el momento en que $\mathrm{Ch}$. Witke, con certeza creemos, ha observado que estas expresiones tienen un carácter retórico y didáctico - según la tradición cristiana iniciada principalmente con S. Pablo-: "Prudentius trouglout the body of this hymn (i. e. el Paristephanon) remains the poet of noster populus... etc.n, cf. Numen Litterarum. The Old and the new in Latin poetry from Constantine to Gregory the Great, Mittellateinische Studien und Texte, Leiden, 197x, p. 136. Sobre Prudencis en general véase Schanz-Hosius, Geschichte der Römischen Literatı̌r, IV r, p. 233 ss. y también el gran estudio de A. Puech, Prudence. Etude sur la poésie chrétienne au $I V$ siècle, Paris, 1888.

2 Praef. 19, 21. Cf. el comentario de PLRE en p. $21_{4}$ a este oscuro pasaje. Witke, op. cit., p. ז20, lo da por hecho.

3 Puech, Prudence, p. 62 ss.

- Schanz-Hosius, Geschichte, pp. 233-236; Witke, Numen, pp. I20 y 121. 
Principibus tamen e cunctis non defuit unus $45^{\circ}$ me puero, ut menini, ductor fortissimus armis, conditor et legum, celeberrimus ore manuque, consultor patriae, sed non consultor habendae religionis, anans ter centum milia diuum. Perfidus ille deo quamuis non perfidus orbi.

455 Augustum caput ante pedes curuare Mineruae, fictilis et soleas Iunonis lambere, plantis Herculis aduolui, genua incerare Dianae, quin et Apollineo frontem submittere gypso aut Pollucis equum suffire ardentibus extis.

460 Forte litans Hecaten placabat sanguine multo. Pontificum festis ferienda securibus illic agmina uaccarum steterant uitulasque reuincta fronte coronatas umbrabat torta cupressus. Iamque insertato reserarat uiscera cultro

465 uittatus de more senex manibusque cruentis tractabat trepidas letali frigore fibras postremosque animae pulsus in corde tepenti callidus interpres numeris et fine notabat, cum subito exclamat media inter sacra sacerdos

470 pallidus: "Lìn, quid ago? Maius, rex optime, maius numen nescio quod nostris interuenit aris quam sufferre queant spumantia cymbia lacte caesarum sanguis pecudum uerbena coronae. Accitas uideo longe dispergier umbras.

475 '́etrita P'ersej,hone uertit nestigia retro cxtinctis facibus tracto fugitiua flagello. Nil agit arcanum murmur, nil Thessala prosunt carmina, turbatos reuocat nulla hostia manes. Noune uides ut turibulis frigentibus iguis

480 marceat, ut canis pigrescat pruma fauillis? Ecce palatinus pateram retinere minister non ualet, elisa destillant balsama dextra, flamen et ipse suas miratur uertice laurus cedere, et incertum frustratur uictima ferrum.

${ }_{4} 85$ Nescio quis certe subrepsit christicolarum hic iutenum; genus hoc hominum tremit infula et omne puluinar diuum. Lotus procul absit et unctus. l'ulchra reformatis redeat l'roserpina sacris?" Dixit et exsanguis conlabitur ac uelut ipsum

490 cerneret exerto minitantem fulmine Christum. Ipse quoque examimis posito diademate princeps pallet, et adstantes circum inspicit ecquis alumnus chrismatis inscripto signaret tempora ligno, qui Zoroastreos turbasset fronte susurros. 
495 Armiger e cunco puerorum flauicomantum, purpurei custos latcris, deprenditur umus nec negat et gemino gemmata hastilia ferro proicit ac signum Cluristi se ferre fatctur. Prosiluit pauidus deiecto antistitc princeps

500 marmoreum fugiens nullo comitante sacellum. dum tremefacta colors donninique oblita supinas erigit ad caelum facies atque inuocat Iesum.

Antes de pasar a analizar el texto en si -análisis en el que no nos vamos a ocupar de los aspectos filológicos- conviene resumir lo que se dice líneas antes.

Prudencio se dirige a Judea y pregunta enfáticamente: Haec, Iudea, tuas uox non perucnit ad aures? (v. 420). La voz del cristianismo llegó -es la contestación del poeta - al pueblo judío pero no hizo mella alguna en él: mentem sed non penctraut egenam / lucis et a primis foribus disclusa refugit (vv. 42I-422). Sin embargo, continúa Prudencio, todos los denís pueblos la han oído ya: ha llegado hasta Iberia (Hispania), a los pueblos del Oriente, a Isscitia, Hircania, el Hebro y el Rodope, hasta el Cáucaso, y los getas y las tribus moras del Atlas han oído hablar del cristianismo (vv. 424-434).

Toda esta enumeración responde a la idea -tópica ya en esta época- de que se cumplen las palabras del Salmo $(18,5)$ que dicen: "Ein toda la tierra resonó la voz y sus palabras llegaron a los confines de la tierras ${ }^{1}$.

Continúa Prudencio diciendo que después de que Dios bajó a la tierra y se hizo hombre ( $\mathrm{r} \cdot 435-436)$, a partir de ese momento el oráculo de Delfos calló y no hubo ya respuestas ${ }^{2}$, ni hulıo tampoco más oráculos sibilinos, ni en Dodona ni en Cumas, etc. (vv. 438-443). El Capitolio de Roma se lamenta de que Cristo sea el Dios de sus príncipes y se entristece también de ver los templos destruidos por orden de los Emperadores. Y sigue diciendo: iam puipura supplex / sternitur. Aeneadce rectoris ad atria Christi / ucxillumquc crucis summus dominator adorat.

La alusión a que el Capitolio se lamente de que Cristo sea el Lirs de los timperadores romanos es una posiljle referencia a la polémica

1 Cf. Diaz y Diaz, "En torno a los origenes del cristianismo hispánico", en Raices de España, Madrid, 1967, p. 434; cf. Ireneo, Ad Haevesiarcas, I 10; Tert11liano, Aduersus Iudacos, 7; Jerónimo, Dial. contra Luc., cap. ${ }_{15}$.

2 II. W. Parke and D. L. W. Wormell, The Delphic Oracle, Oxford, 1956, pp. 288-9 (vol. I), amune el oráculo conoció una revitalización bajo Adriano. 
surgicla a propósito del incidente del altar de la Victoria ${ }^{1}$; y la mención de los templos destruidos es una exageración en gran medida ${ }^{2}$. Antes de Juliano $y$ desde Constantino, los ucrilla de las legiones portaban el labarum; Juliano lo sustituyó por la representación de los dioses Y más tarde, con Joviano, volvió a imponerse el labarum ${ }^{3}$. De aquí la alusión a que los Emperadores se postren ante ellos. Fin estos versos que preceden a los que vamos a comentar, el poeta ha trazado admirablemente todas las principales características de la propaganda cristiana y de su victoria definitiva sobre el paganismo. Prudencio se está refiriendo a la época de los Emperadores posteriores a Constantino; por ello, a continuación, introduce el episodio de Juliano: Principibus tamen e cunctis non defuit unus, etc. (v. 449).

En esta especie de excursus sobre el único Emperador pagano del siglo IV se distinguen claramente dos partes: la primera integrada por los versos 449-454 y la segunda desde 455 hasta el fin.

En la primera Prudencio hace una rápida semblanza o retrato de Juliano que es $\mathrm{y}$ obedece a las leyes de panegírico casi estrictamente (uid. infra), aunque insiste con gran énfasis en el paganismo del Eimperador. En la segunda relata un milagro que sucedió estando Juliano sacrificando. La finalidad de esta anécdota es demostrar que, aunque Juliano fue una excepción dentro de los Emperadores que aceptaron el Cristianismo, no por eso se sustrajo a ser vencido y dominado por él,

1 Iis, al menos, una posibilidad. Cf. O. Seeck, Q. Aurelii Symmachi quae supersunt, Berlin, 1883 (reimpr. 1961), p. LXXII, donde habla de un viaje a Milán de Simmaco en 402 , ocasión en la que, una vez más, tendría oportunidad de hablar del asunto. Algunos autores defienden la posibilidad de esta última intervención aunque, recientemente, F. Paschoud la descarta en Roma Aeterna. Etudes sur le patriotisme romain dans l'Occident a l'époque des grandes invasions, Neuchâtel, 1967, pp. 223-224. El propio Paschoud adelantaba esta afirmación en "Reflexions sur l'ideal religieux de Symmaque", Historia, 14, 1965, p. 218 con n. I9 (en general todo este artículo es fundamental para el problema de la polémica del Altar de la Victoria).

2 Más que destrucción de templos hubo un sistemático expolio de muchos de ellos. He hecho alusión a este problema en un trabajo sobre "Las reconstrucciones de templos paganos en época del Emperador Juliano", Rivista Storica dill' Antichitò (en prensa). Siguió habiendo reconstrucciones y dedicaciones de templos hasta muy avanzado el s. IV, como lo demuestra la estadistica hecha por Salama para Africa en Acta of the Fith International Congress of Greek and Latin Epigraphy. Cambridge 1967, Oxford, 1971, pp. 285-6. Sobre lo mismo véase también Prudencio Contra Symm. I 501-5.

3 Cf. G. Elmer, "Die Kupfergeldreform unter Julianus Philosophus", NZ, 1937, p. 4r y A. Piganiol, L'Empire Chrétien, Paris, 1945, p. 298. 
con to cual Prudencio enlaza justamente con los versos anteriores (v. 444 ss.). De aquí que, creemos, se extienda un poco excesivamente en el relato detallado de lo ocurrido, relato que constituye al mismo tiempo un aviso y una enseñanza. A la vez, este detenerse en el episodio de Juliano da clara muestra de la gran conmoción que éste causó en sus contemporáneos: Juliano había penetrado ya en la leyenda y constituía un buen ejemplo para llamar la atención a posibles initadores.

En v. 450 ss. dice Prudericio que recuerda (memini) que siendo niño (me puero) hubo un Emperador que no fue como el resto. De él había oído Prudencio, o leído, por un lado su capacidad como militar y estratega (ductor fortissimus armis); de su actividad legislativa brillante (conditor et legum); de su elocuencia y de su valor (celeberrimus ore manuque); de su preocupación por el Imperio (consultor patriae). Pero había oído o leído mucho más acerca de su dedicación a los dioses paganos: sed non consultor habendae / religionis, amans ter centum milia dinum. $\mathrm{Y}$ por fin, el verso $45+$ expresa la propia opinión de Prudencio sobre el Emperador: Perfidus ille deo, quamuis non perfidus orbi.

Algunos autores han querido deducir del ut memini que Prudencio en su niñez vivió en un ambiente cristiano e incluso que fue bautizado, porque el verbo expresaría la idea de que sienclo niño escucharía de labios de sus padres historias sobre el pérfido Juliano como la que relata a continuación ${ }^{1}$. La deducción es excesiva (al margen de que Prudencio de hecho haya pertenecido a una familia cristiana), ya que el ut memini puede referirse también a lecturas o, por el contrario, la historia pudo haber sido comentada en un ambiente pagano.

La expresión me puero proporciona una cierta indicación cronológica sobre el cuándo tuvo lugar el acontecimiento narrado en el poema. Si tenemos en cuenta que el poeta nació en el $34^{8}$ (cf. supra n. 3 , p. I3I) y si nos atenemos a que Juliano aparece en los versos sacrificando como pontifex maximus ante su ejército (cf. v. 50r, donde se habla de una cohorte que presenciaba el sacrificio: dum tremefacta cohors, etc.), y si consideramos que esta actividad sacrificadora se intensifica durante la estancia en Antioquía y que el texto parece coincidir con la descripción de Amiano XXII I2,6 que dice: Hostiarum tamen sanguine plurimo

1 Asi J. Guillén en Prudencio. Obras Completas, Madrid, I950, p. 4. 
aras crebritate nimia porfundebat, tauros aliquotiens immolando centenos et inmumeros uarii pecoris greges... adeo ut in dies paene singulos milites carnis distentiore sagina... ad sua diuersoria portarentur. Petulantes ante omnes et Celtae, quorum ea tempestate confidentia creuerat ultra modum ${ }^{2}$ (obsérvese que en la descripción de Prudencio se habla de flanicomantim posiblemente componentes de su tropa germánica), se puede pensar que el acontecimiento tendría lugar entre mediados del 362 y $363^{2}$ y por lo tanto Prudencio tendría entonces I4 años.

Prudencio llama a Juliano ductor fortissimus armis (v. 450). F,1 historiador Zósimo, escribiendo entre 50r-5IS, dice que en el sarcófago de Juliano había una inscripción en la yue se léia una alabanza se-

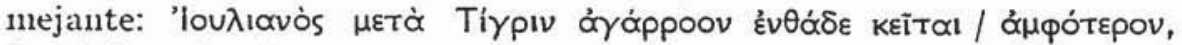

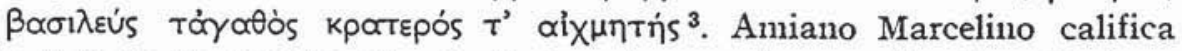
a Juliano de armatac rei scicntissimus ${ }^{4}$ y en otra ocasión dice: Castrensium negotiorum scientiam plura declarant et nota (Amm. XXV 4, II); incluso se ha pensado que Juliano sería el autor de un tratado sobre armas de guerra titulado $\mu \eta \chi \propto \propto v ı \kappa \alpha ́$. Los epitomistas Aurelio Víctor ${ }^{5}$ y el autor del Epitome de Caesaribus (obra escrita en Roma hacia el 400) alaban sus dotes militares en la Galia y en Persia ${ }^{6}$, así como Libanio lo hace también repetidamente? ${ }^{7}$ De hecho las campañas de Juliano en las Galias - con la brillante victoria de Argentoratumy la genial concepción de la campaña de Persia, hacen de él un estratega extraordinario reconocido por todos ${ }^{8}$. Por estas dotes obtuvo

1 Am. Marc. XXII 12, 6.

2 Juliano entró en Antioquía el 19 de julio de 362, cf. J. Bidez, Lettres de l'Empereur Julien, Paris, 1960, p. 79 y 92 ss.

3 Zos. III $34 ;$ Ant. Pal. VII 747; W. Pcek, Griechische Grabgedichte, Berlin, I960, n. 377; discusión sobre el epitafio en nuestra tesis doctoral Estudios sobre las fusentes literarias, epigraficas y numismáticas para la historia del Emperador Juliano;además Ph. Grierson, "The Tombs and Obits of the Byzantine Emperors", Dumbarton Oaks Papers, 16, 1962, p. 40.

4 An. Marc. $\mathrm{XXV}_{4}, 7$. Que Amiano diga esto, él que conocía intimamente todos los problemas de la milicia (recuérdese el ut miles quondam et graecus), tiene un gran valor. Además $\mathrm{XXV}_{4}$, ro-II.

5 XLII I7 (ed. Pilchmayr).

6 XLII I3.

7 Lib., Orat. XVIII 63 ss. y 282.

8 Cf. Bidez, La Vie, 1. 149; Barbagallo en Dizionario Epigrafico, s. u. Iulianus; Piganiol, L'Empire Chrétien, p. I43, que destaca especialmente las campañas de las Galias, aunque no creo que tenga razón el reprocharle que la combinación pretendicla con Procopio estuvo mal organizacla. Nos gustaria saber por qué el cuerpo de ejército de Procopio no logró reunirse con el de Juliano. La acción 
los títulos de Germánico, Alamánico y Fráncico y se dejó llamar Sarmático ${ }^{1}$, aspirando, en el decir de Amiano, al de Pártico ${ }^{2}$.

La segunda referencia de Prudencio a Juliano alude a su capacidad de legislador y administrador: conditor et legum. También en esto Ammiano coincide con Prudencio al afirmar: studiosus cognitionum omnium et indeclinabilis aliquotiens iudex; censor in moribus referendis acerrimus... quibus autem iustitiae inclarnit bonis, multa significant, primo quod erat pro rerum et hominum distinctione, sine crudelitate terribilis, etc. ${ }^{31}$ ( $\mathrm{y}$ véase también $\mathrm{XXV} 4,20$ ). Libanio insiste en este aspecto en muchas ocasiones ${ }^{4}$. Varias de estas leyes, recogidas en el Codex Theodosianus ${ }^{5} \mathrm{y}$ algunas cn inscripciones ${ }^{6}$, dan testimonio de esta actividacl, siempre atenta al bienestar de los súbditos, y que incluso los cristianos se vieron obligados a ensalzar ?

Prudencio hace alusión, cómo no, a su cultura extraordinaria y a su valor: celeberrimus ore manuque. E1 epitomista Eutropio, pagano y que participó en la campaña persa, había dicho de él: liberalis disciplinis adprime cruditus, graecis doctior atque adeo ut latina eruditio nequaquam cum Graeca scientia conueniret, facundia ingenti et prompta, memoriae tenacissimae, in quibusdam philosopho propior ${ }^{8}$. Amiano hace también alusión a la constante actividad intelectual de $\mathrm{Juliano}^{9} \mathrm{y}$ el autor del Epitome señala: Fucrat in eo litterarum ac negotiorum ingens scientia,

estaba muy bien pensada si se tiene en cuenta que mediante dicha combinación se pretendia atenazar a los persas. P. Allard pretendió igualmente desacreditar a Juliano por el fracaso de la campaña. Sin embargo, véase la reinvindicación acertada de R. T. Ridley en "Notes on Julian's Persian Expedition (363)", Historia, 22, 1973, p. 317 ss. especialmente pp. 325-326.

1 Cf. Jalabert, Mél. Fac. Univ. St. Joseph, Beyrouth, r9o7, p. $26 j$ ss.; IL.S $8945=C I L$ III I2.333; A. Negev, Israel Exploration Journal, I969, p. I70; J. Marcillet-Jaubert, "Dedicace à Julien", en Salamine de Chipre. IV. Anthologie Salaminiene, Paris, 1973, pp. I21-123; V. Beseliev, Spätgriechische und Spätlateinische. Inschriften aus Bulgarien, Berlin, 1964, n. 2 . Soìre el problema del uso indebido del titulo Sarmático hemos tratado en nuestra Tesis ampliamente.

2 Ornamentis illustrium gloriarum inserere Parthici cognomen tum ardebat (XXII I2,2)

3 Am. Marc. XXV 4,7 y 8.

4 Lib., Orat. XVIII 82 ss., 130 ss. y $15 x$ ss.

5 Bidez-Cumont, Iuliani Imperatoris Epistulae Leges Poematia Fragmenta Varia, Paris-Londres, 1922 , passim. (ELF).

$6 E L F$, n. 68, p. $80=I L S 755$ y $C I L$, V 8.987.

7 Greg. Naz. Orat. IV 75.

$8 \mathrm{X} 16,3$.

- $\mathrm{XXV}_{4}, 5 \mathrm{y} 6$. 
aequauerat philosophos et graecortum sapientissimos ${ }^{1}$. Las inscripciones 1o recuerdan igualmente como un Emperador-filósofo ${ }^{2}$ y los autores cristianos dan constancia tanto de su decidida intención de gobernar como tal, como de su actividad literaria ${ }^{3}$. Libanio, en fin, se adnira repetidamente de la elocuencia de su discípulo (cf. Orat. I I30 y XVIII I5) y alaba sus conocimientos 4 . En cuanto al valor, Amiano destaca que frecuentemente iba él mismo al frente de sus ejércitos y participaba en los combates como uno más (cf. XXV 4, Io y II) ${ }^{5}$.

En el consultor patriae se vienen a resumir todas las virtudes de Juliano. Eutropio lo había llamado rem publicam insigniter moderaturus y había dicho de él que fue in prouinciales iustissimus ct tributormm quatcnus fieri possct repressor ${ }^{6}$. En las inscripciones y en autores como I.ibanio y Amiano se repiten constantemente los ejemplos del interés de Juliano por el Imperio y se hacen alusiones a sus beneficiosas reformas tanto en las Galias como en otras provincias?.

Después de todas estas alabanzas Prudencio hace un único reproche al Emperador: sed non consultor habendae/religionis, amans ter centum milia diunm. Es natural que el poeta cristiano tenga este reproche para el Emperador que más inteligentemente luchó por acabar con la religión de «los galileos». Prudencio, además, pensaba que "no hay incompatibilidad fundamental entre los deberes hacia el Estado y los deberes hacia la Iglesia y que jamás las exigencias divinas impidieron a un hombre realizar su propia obra en la tierra", como recientemente ha destacado F. Paschoud ${ }^{8}$.

Es curioso notar que todos los autores paganos, excepto Libanio, hablan en un sentido idéntico al de Prudencio por lo que se refiere a la religión de Juliano: Amiano, en concreto, dice casi las mismas pala-

1 XLIII 5.

2 Cf. Diehl en $B C H$, 1889, p. 35; Doro Levi, Ann. Sch. Arch. Ath., 1963, página 580, fig. 8; ILS $75 \mathrm{I}=C I L$, III 7.088; Mamertino, Gratiarum Actio, 23, 4 .

3 Soc. $H E$, III $\mathbf{x}$.

- Lib., Orat. XVIII 301-303, cf. también I6r y 284 , etc.

5 Un ejemplo de ello seria el hecho de que Juliano murió precisamente por haberse puesto al frente de un grupo de soldados para repeler un repentino ataque persa sin escudo y prácticamente desarmado.

B $\mathrm{X} 16,3$.

7 CIL IX 5.960; 4r7; XI 6.669; V 8.987; Am. Marc. XXV 4, I5; XVI 5 , $\mathrm{I}_{5} ; \mathrm{XX} 5,7$; A. Andreotti, Nuova Rivista Storica, I4, 1930, p. 345; J. Geffcken, Kaiser Julian, Leipzig, I9II, p. 69 ss.; E. Condurachi, "La politique financière de l'Empereur Julian", Acad. Roum. Bull. de la Sec. Hist., 22, 1941, p. 102 ss.

8 Paschoud, Roma Aeterna, p. 227; Prud., Contra Symm. I 620-23. 
bras: superstitiosus magis quam sacrorum legitimus obseruator, inmensas sine parsimonia pecudes mactans, ut aestimaretur (si renertisset de Parthis) boues iam defuturos ${ }^{1}$. El autor del Epitome lo califica como cultus numinum superstitiosus ${ }^{2}$ y Eutropio observa: religionis Christianae insectator ac perinde tamen ut cruore abstineret ${ }^{3}$.

Ira opinión final de Prudencio se expresa, como hemos dicho, en la famosa frase: Perfidus ille deo, sed non perfidus orbi.

El vocablo perfidus está especialmente bien elegido por cuanto que el sistema de persecución de Juliano no fue violento, por ejemplo, como el de Diocleciano, sino mucho más sutil ${ }^{4}$. Orbi significa aquí el Imperio Romano en gencral y la historiografía moderna está de acuerdo en reconocer y constatar que efectivamente el gobierno de Juliano, aunque corto, significó y fue auténtica expresión de un intento de remediar muchos de los problemas del Imperio y que en la medida de lo posible fue muy beneficioso ${ }^{5}$.

En estos versos Prudencio ha demostrado que su patriotismo romano está por encima de subjetividades y aun de ideologías y, como ha señalado también F. Paschoud, da muestra "d'une admirable largeur de vues et d'une louable tolerance" ${ }^{6}$.

En pocas palabras el poeta ha trazado un verdadero panegírico equiparable en cierto modo al de Amiano Marcellino, hasta el punto de que nos atreveríamos a sugerir una inspiración directa bien en Amiano, bien en Eutropio. Amiano, antes de exponer los vicios y virtudes de Juliano, señaló: cum enim sint (ut sapientes definiunt) uirtutes qualtuor praecipuae, temperantia, prudentia, iustitia, fortitudo, eisque accedentes extrinsecae aliae, scientia rei militaris, auctoritas, felicitas atque liberalitas intento studio coluit (sc. Iulianus) omnes ut singulas (XXV 4, I). Prudencio, aunque no de forma sistemática, ha hecho referencia a casi todas ellas: prudentia (conditor patriae); iustitia (conditor legum); fortitudo (celeberrimus ore manuque); scientia rei militaris (ductor fortissimus armis).

Ningún autor cristiano ha hablado de Juliano con más veracidad ni más objetividad. Prudencio está muy lejos de las sañudas invectivas

$1 \mathrm{XXV}_{4}, 17$.

2 XLIII 7.

3 Eutr. X 16, 3; Orosio, Ad. Pag. (ed. Zangemeister), VII 30, 2.

4 Soc., HE. III 12.

5 Baste con mencionar aqui los juicios de S. Mazzarino en Trattato di Storia di Roma, p. 463 ss.; del mismo autor en Aspetti Sociali del IV Secolo, Roma, r95I, p. I 10 ss. y de Piganiol, L'Empire Chrétien, pp. I43-45, aunque discutible.

- Paschoud, Roma Aeterna, p. 227. 
de un Gregorio de Nazianzo y se encuentra, en cambio, mucho más cerca de la historiografía pagana que curiosamente, y quizás debido a condicionamientos políticos contemporáneos, no dejó de constatar con cierto aire de lamentación el hecho de que Juliano fuera excesivamente dado al paganismo y a los sacrificios. De una manera general se puede decir que la historiografía oriental es mucho más detractora y fanática respecto a Juliano, mientras que la occidental es más ecuánime ${ }^{1}$.

Los versos 460-502 constituyen la narración de un milagro que sucedió estando saćrificando el Emperador. El flamen hace esfuerzos desesperados para obtener un correcto ritual; pero todo es inútil. Pregunta entonces si entre los presentes se encuentra algún cristiano: Nescio quis certe subrepsit christicolarum / hic iunenum; genus hoc hominum tremit infula et omne / puluinar diunm (vv. 485-87). Juliano palidece y se inicia una investigación que da como resultado que efectivamente uno de los soldados asistentes reconoce llevar encima el signo de Cristo. Juliano se retira entonces en silencio. La cohorte, atemorizada, y en vista de que ya no está el Ėmperador presente, comienza una plegaria a Jesís.

El pasaje tiene un antecedente paralelo en un párrafo de Lactancio hablando de Diocleciano: Tum quidam ministrorum scientes dominum cum adsistercnt inmolanti, imposuerunt frontibus suis inmortale signum: quo facto fugatis daemonibus sacra turbata sunt. Trepidabant aruspices nec solitos in extis notos uidebant et quasi non litassent saepius inmolabant. Verum identidem mactatae hostiae nihil oslendebant, donec magister ille aruspicum Tagis seu suspicione seu uisu ait idcirco non respondere sacra quod rebus diuinis profani homines interessent. Tunc ira furens sacrificare non eos tantum qui sacris ministrabant, sed uniutersos, etc. ${ }^{2}$. Se trata evidentemente de una situación tópica creada literariamente en época anterior a Prudencio pero que éste supo combinar perfectamente con otros elementos aplicados a Juliano. Porque sabemos por otros historia-

1 La acción de Inliano en Oriente fue mucho más intensa que cn Occidente (prácticamente pasó todo su gohierno alli). Hay que pensar también en que esta reacción es (lebida al auge enorme del cristianisuno en esa zona y al carácter polemista de los autores cristianos de la época.

2 De mortibus persecutoiun, 10, 2-4 (ed. J. Noreau); para más referencias cf. el vol. Il de notas de la edición de Moreau, p. 264. 
dores que el limperador, sobre todo con motivo de la campaña de Persia, consultaba frecuentemente las entrañas de los animales para intentar deducir un presagio favorable ${ }^{1}$. En esta ocasión se trataba de un sacrificio a Hécate, aunque algunos elementos y alusiones nos hacen pensar que puede tratarse de un culto relacionado con el dios Mitra ${ }^{2}$.

Es importante notar que, según se desprende del texto de Prudencio, en el ejército de Juliano se mantenían cristianos en secreto, a pesar de las leyes promulgadas por el Emperador al respecto ${ }^{3}$. Al parecer, incluso, todos los componentes de la cohorte eran cristianos, pero mantenían su religión oculta por temor ${ }^{4}$.

Los versos de Prudencio sobre Juliano son un documento preciso, exacto y de gran valor histórico por su objetividad refrendada por múltiples testimonios paralelos contemporáneos de diversa tendencia y naturaleza. Lástima que la historiografia cristiana posterior se haya fijado al hablar de Juliano fundamentalmente en lo anecdótico.

J. ARCE

1 Am. Marc. XXIV 6, 17; XXV 2, 7. Cf. G. Downey, History of Antioch in Syria, Princeton, 1961, p. $3^{84}$ ss. y D. Concluché, "Ammien Marcellin et la mort de Julicnn, Latomus, 24 , 1965, p. 359 ss., especialmente 368 y 370.

2 Lil verso 494 qui Zoroastreos turbasset fronte susturros parece una alusión a los himnos que está atestiguado se recitaljan en los sacrificios mitraicos. Véase M. J. Vermascren, Mithras, the secret God, London, I963, p. I68 ss. La Iresencia de soldados en el sacrificio y la propia arraigada devoción al dios por parte de Juliano nos inclinan a pensar asi. No obstante, en el pasaje Prudencio mezcla un tanto arbitrariamente todos los elementos que le parecen relevantes y tipicos de un sacrificio pagano sin importarle mucho la exactitud del hecho en si.

3 Cf. Rufino de Aquilea, $H E, X$ 33; Soc. III I3; Juan Antioqueno, fr. I 79 . Se ha entablado una discusión en torno a si la ley afectó sólo a los militares de rango superior o a todos en general, problema que hemos tratado en "Los cambios en la administración imperial y provincial con el Emperador Juliano", Hispania Antiqua, 5 (en prensa).

4 Dum tremefacta cohors dominique oblita supinas / erigit ad caelntm facies atque inuocat Hisum (vv. 501-502). 